\title{
What's in a Cluster? \\ Automatically Detecting Interesting Interactions in Student E-Discussions
}

\author{
Jan Miksatko and Bruce M. McLaren \\ Deutsches Forschungszentrum für Künstliche Intelligenz (DFKI) \\ Stuhlsatzenhausweg 3 \\ D-66123 Saarbrücken Germany \\ \{honza.miksatko,bmclaren\}@dfki.de
}

\begin{abstract}
Students in classrooms are starting to use visual argumentation tools for e-discussions - a form of debate in which contributions are written into graphical shapes and linked to one another according to whether they, for instance, support or oppose one another. In order to moderate several simultaneous e-discussions effectively, teachers must be alerted regarding events of interest. We focused on the identification of clusters of contributions representing interaction patterns that are of pedagogical interest (e.g., a student clarifies his or her opinion and then gets feedback from other students). We designed an algorithm that takes an example cluster as input and uses inexact graph matching, text analysis, and machine learning classifiers to search for similar patterns in a given corpus. The method was evaluated on an annotated dataset of real ediscussions and was able to detect almost $80 \%$ of the annotated clusters while providing acceptable precision performance.
\end{abstract}

Keywords: Educational Data mining, Machine Learning in ITS, Collaborative Learning, Natural Language and Discourse.

\section{Introduction}

One of the important trends in Computer-Supported Collaborative Learning (CSCL) is the development and use of networked visual argumentation tools that allow students to work on separate computers and express their ideas, questions, and arguments in visual fashion. Students make contributions to the online discussion by dragging and dropping shapes with different meanings (e.g. "claim" or "question"), filling them with text containing their contributions to the discussion, and linking the shapes to other relevant shapes with labeled links, such as "opposes" or "supports." An example of such an e-discussion in the Digalo collaboration software is shown in Fig. 1 (text in the shapes shows only the title of the contribution).

Although computer-based tools for collaboration, argumentation, and discussion are becoming relatively commonplace in schools [1,2], there is a critical need for software that can help teachers observe, guide, and moderate such e-discussions. For instance, suppose a classroom of students, organized in small discussion groups of 4 
to 6 students, is tasked with discussing and debating a social sciences topic such as "Is it ethical to perform experiments on animals?" using a visual collaboration tool. The teacher in such a classroom obviously cannot monitor and moderate all of these discussions simultaneously without some automated system support. Furthermore, past research suggests that discussion and collaboration tools used by students on their own with no support does not typically lead to fruitful collaboration [3].

In our work on the ARGUNAUT project [4], the Moderator's Interface (MI) - a software tool that displays multiple simultaneous e-discussions taking place in the classroom - provides the teacher with such support by pointing her to events requiring human intervention.

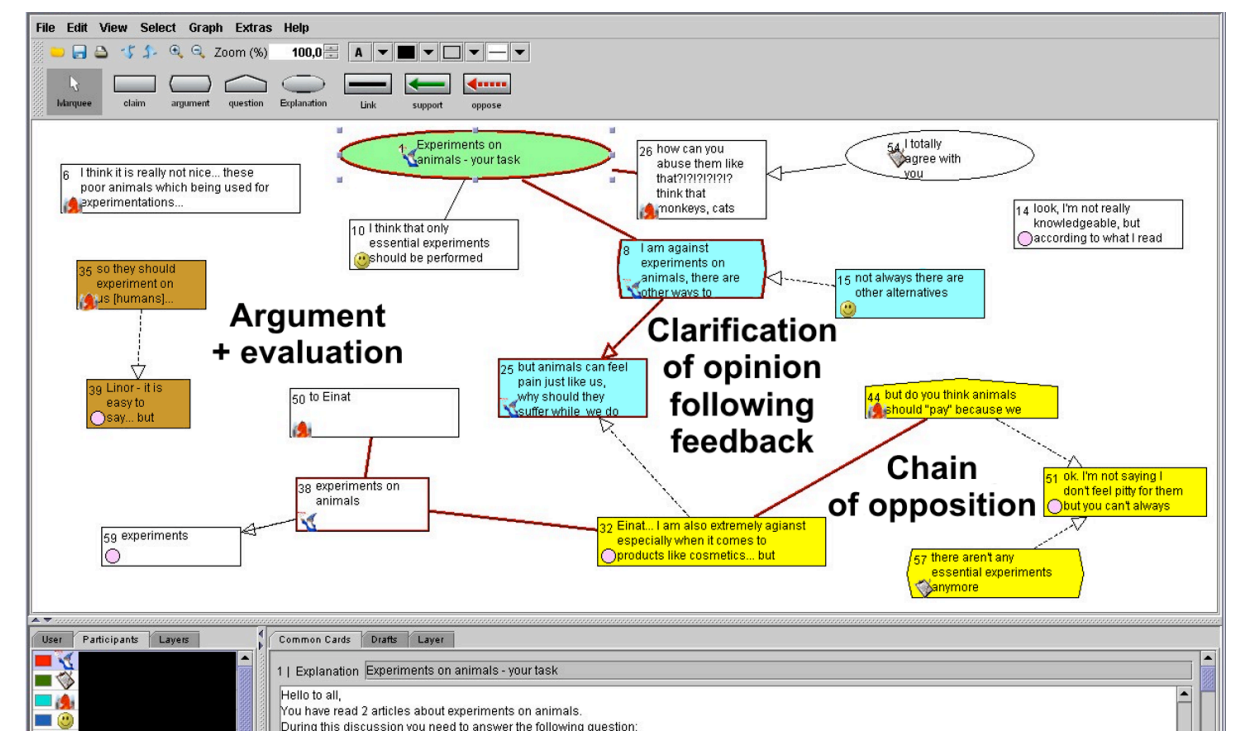

Fig. 1. A well-structured discussion in Digalo software with three simple clusters ${ }^{1}$

In the present work, we are trying to address the problem of identifying complex interaction patterns in the e-discussions. Such patterns, called clusters in the remainder of the paper, are multiple contributions, typically (but not exclusively) made by different students, that capture interesting interactions in the e-discussion. Fig. 1 shows an example of a few such clusters. For instance, "Clarification of opinion following feedback" involves a student clarifying his or her opinion and then getting feedback from other students. Types of clusters representing interesting interactions are specified and annotated by the pedagogical researchers on the ARGUNAUT project, with an eye toward moderating e-discussions. Our primary aim is to provide teachers, the users of the MI, with a tool that can point them to interesting conversational moves and clusters in the discussions [5]. A secondary goal is to support the pedagogical researchers in searching off-line for interesting patterns, as they evaluate and data mine past discussions.

\footnotetext{
${ }^{1}$ The names of students in this discussion have been anonymized to protect their identities.
} 
Our task is a daunting one because

(1) we are dealing with highly complex data (i.e., a combination of graph structure and text),

(2) discussion "maps" (as they will be called henceforth) typically have quite a bit of noisy data,

(3) cluster types are difficult to precisely specify, and

(4) we have a limited source of annotated data, since annotating clusters in real discussions is extremely time-consuming and difficult.

We explored several approaches but ultimately designed and developed one approach that seemed to best fit the problem characteristics above: DOCE (Detection of $\boldsymbol{C}$ lusters by $\boldsymbol{E}$ xample). DOCE is based on the idea of using cluster examples to find similar clusters in other discussions and has demonstrated very promising preliminary results on an initial set of annotated maps. The main advantages of the DOCE algorithm can be summarized as follows:

- The algorithm does not require precisely defined clusters; instead, it employs an intuitive approach in which cluster examples are provided.

- Only a few annotations are required, as examples for queries, contrary to the large number of examples required by supervised methods. Furthermore, it provides a tool for collecting the annotations.

- It can detect clusters based on their structural and content features, important to the goals of the ARGUNAUT project.

- It is noise tolerant, as it looks for similar, not exactly the same, clusters.

In this paper we describe the DOCE algorithm and present our initial, encouraging results.

\section{Related Work}

Analyzing student contributions and assigning labels is common practice in designing and experimenting with intelligent educational technology. For instance, the researchers in [6] investigated machine-learning approaches by training classifiers on the language of a large corpus of labeled data and classifying single contributions into categories. These results led to the development of TagHelper - a tool for text classification that is also utilized in our work.

In addition to the text classification capability of TagHelper, our work with DOCE also incorporates the structure of the discussion by using machine-learned classifications of single contributions (e.g. Topic Focus, Reasoned Claim) and paired contributions (e.g. Contribution-Supporting Argument) [5,7]. Contributions are characterized by a combination of text features extracted by TagHelper and structural attributes relevant to the e-discussions, such as shape type and number of in- and out- links. Several highly reliable classifiers (with Kappa $>0.6$ ) have been trained and integrated in the Moderator's Interface as "Awareness Indicators", as discussed in [5,7].

However, such supervised learning approaches do not scale well to clusters of arbitrary size. Clusters not only need to be classified, as in standard machine learning approaches, but also recognized in the discussion. In addition, obtaining a sufficient 
number of annotations for training at the cluster level is a very time consuming and difficult task - much more difficult than annotating single and paired contributions.

A related unsupervised method, detection of frequently reoccurring patterns, was applied in [8] for identifying common interaction patterns during student software development projects on data from source repository logs or Wiki pages. However, the clusters defined by our expert annotaters do not necessarily occur as frequently in our domain as in theirs. A similar approach was evaluated on the ARGUNAUT project in [9]. A tool was designed for mining sequences of actions in the discussions, such as "create shape"/"add link"/"modify text." The tool was able to detect some commonly occurring patterns. However, their exact-matching algorithm was unable to detect all of the patterns of given cluster types, especially when clusters differed in subtle and imprecise ways from one another. Our goal was to address these issues with DOCE.

\section{Detection of Clusters by Example (DOCE)}

The DOCE algorithm is based on the Query By Example (QBE) technique that has been applied to databases as a query method. The idea is to search for similar files or documents based on an input example: a text string, a document, or visual table example [10]. The AI subfield of case-based reasoning [11] is another research area in which examples (i.e., cases) are used to search for similar instances in a repository of data (i.e., a "case base"). A teacher or researcher selects a cluster (e.g. connected individual contributions) in an existing discussion that exemplifies an interesting pattern. The example cluster (also called a "model graph" in the following text) is then used as a search query for similar clusters across other discussion maps (called "input graphs"). The output of the algorithm is a list of matching clusters in the discussion map(s), sorted according to a similarity rating. DOCE can be used as a "live" classifier of clusters - characteristic example(s) representing a cluster of a particular type are stored in the database and used later as queries for automated cluster detection. Or, it can be used as a research tool for obtaining clusters and annotating them in the first place.

The DOCE algorithm is sketched in Fig. 2. First, the example cluster and the discussion map are parsed from an XML file format that is used by the Moderator's Interface for representing a snapshot of the discussion. Both graphs are preprocessed as follows: (1) an adjacency matrix representing the structure of the graph is constructed; (2) each contribution and link in the discussion graph is characterized by a feature vector that is extracted from the attributes associated with the discussion vertex and edge such as shape/link type, text length, link direction and whether the same user created two linked shapes. TagHelper [6] further enriches the feature vectors with additional information from the text analysis of contributions. It performs text processing (e.g. stemming) and extracts textual attributes such as unigrams and bigrams (single words and pairs of words occurring in the text), punctuation (indicator of question or mood of the author) and contains non-stop words (a value predicting if the text is meaningful or not). Additionally, we extend the feature vectors of shapes (links) with the high-accuracy output of shape (pair-shape) classifiers that assign contributions (pairs of contributions, respectively) into categories [5,7]. In the next step, DOCE 
compares the feature vectors of vertices/edges in the model and input graphs by calculating their distance in a manner similar to unsupervised learning algorithms. The proximity is pre-computed for each pair of model/input objects and stored in the similarity matrices.

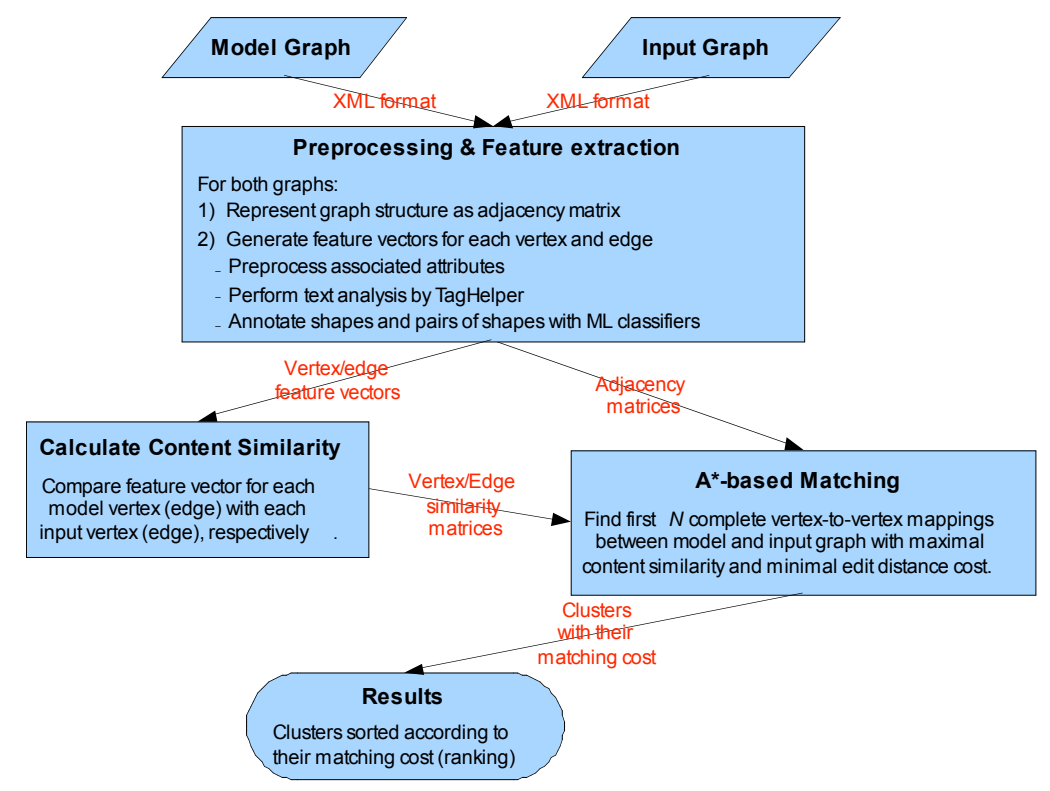

Fig. 2. The DOCE algorithm

Finally, an inexact graph matching method based on a customized version of the edit distance algorithm $[12,13]$ is employed to find clusters with the highest structural and content similarity to the model graph. Similar algorithms have been used for various purposes, such as computer vision [16], pattern recognition [14], and retrieving relevant principles from ethics cases [15]. For instance, in [15] engineering ethics cases and principles were represented in a stylized, graphical language. An undecided case was then matched against past cases and a human was provided with suggestions in deciding the current case.

The matching works as follows. An $A^{*}$ search algorithm explores all possible vertex-to-vertex mappings between the model and input graph. In each step, a partial mapping of vertices is extended by adding a new vertex-to-vertex assignment that has the maximum content similarity (pre-computed in the similarity matrices) and the minimum structural difference, as measured by edit distance. The edit distance between partially matched graphs is calculated as a minimal sequence of primitive graph operations (such as "add an edge", "delete an edge", "delete a vertex") that are required in order to make the graphs isomorphic. The final matching cost is the sum of all vertex/edge similarities and penalties for the edit operations. The first $n$ complete mappings (i.e. mappings that cover all model vertices) are returned as resulting clusters and sorted in ascending matching-cost order.

Thus, the algorithm matches similar clusters on generic graph structures in an inexact manner (e.g., some of our cluster examples are unconnected as well as shapes in 
the discussion may be unlinked). The matching is driven by both the graph structure and content of contributions, for example, the text of the contribution, the users involved in the cluster, and shape type. Note that the detection of all subgraphs is an NP-Complete problem but only in theoretical, not practical, terms. The search space is significantly reduced by applying heuristics similar to $[13,16]$ and the method performs well on graphs of moderate size (dozens of vertices). The graphs in our particular domain are certainly within this range.

The DOCE algorithm is described in further detail in [17].

\section{Evaluation}

We designed an evaluation methodology in which the pedagogical specialists analyzed 27 discussion maps and annotated cluster examples (referred to as "annotations" henceforth) for the three most important types of clusters (as suggested by pedagogical specialists): Clarification of opinion following feedback, Chain of opposition, Argument + evaluation. There were a total of 74 annotations. We used the annotations in each map as input to the DOCE algorithm to evaluate how well the algorithm could find the cluster examples in the other 26 annotated maps. The clusters detected by DOCE were then compared to the annotations in the maps. We compared the performance of DOCE using different feature sets of the algorithm and also compared it to a random algorithm, as there is no other comparable "gold standard" algorithm, at least not for the particular type of problem we are tackling in this work.

Our methodology is similar to Information Retrieval (IR) evaluations - the "Top10" results are considered in the evaluation and the relevancy of results is defined based on user feedback [18]. As already explained, DOCE does not always match clusters in an exact manner. Thus a matching cluster was considered "relevant" if the overlap of vertices between the matching cluster and an annotation is at least 70 $\%$ (rounded) of the annotation size (e.g. if an annotated cluster is $\{1,2,3,4\}$, then a "matched" cluster $\{2,3,4,5\}$ is relevant). The pedagogical experts verified the acceptability of this definition of relevance, which is based on the idea that even a non-exact match can be valuable since the ultimate objective of DOCE is to draw a teacher's attention to interesting behavior in a discussion map, not perfectly match that behavior.

We used several metrics in our evaluation:

- Recall represents the number of relevant matches in the Top10 divided by the count of annotations in the searched map.

- Precision is the number of relevant matches in the Top10 divided by 10.

- Ranking Quality, known as Average Precision in IR, measures the quality of the ordering of the results. A higher value means better ordering of the matching clusters, with the best value being 1.0 (all matches are on at the top of the list).

- Stability is used to evaluate the consistency of the DOCE algorithm with different input models of the same cluster type against the same map. It is calculated as the average intersection size (ranging from 0 to 10) of all pair wise result sets.

We consider Recall to be the most important metric, as it is highly critical to find all of the interesting clusters in a given discussion. We believe the number of relevant 
matches (i.e. Precision) has somewhat lower importance since humans are typically clever enough to filter out irrelevant matches.

\section{Results}

Our overall results, averaged across all models and maps, are presented in Table 1 along with a comparison of different configurations of the algorithm and the Random Matcher. The DOCE Baseline feature set includes only attributes directly available from the structure of the discussion map (e.g. shape type, link direction, users involved in the cluster), with no text considered. The Text configuration includes annotations from the shape/pair-shape level analysis [5,7] and attributes obtained from the TagHelper linguistic analysis [6]. The weights are set to prefer the text attributes. The Text configuration was experimentally chosen as the best combination of features and attribute weights. The parameter $\pi$ (ranging from 0 to 100) influences the ordering and balance of the content similarity and edit operations - high (low) values prefer matches with few (many) edit operations at the top positions in the result list, respectively. We present the results with a "neutral" $\pi$ value $(\pi=50)$ in order to avoid bias from parameter choice, and the manually tuned value $(\pi=100)$.

Table 1. Overall results and comparison of DOCE algorithm to the Random Matcher

\begin{tabular}{|l|c|c|c|c|}
\hline \multicolumn{1}{|c|}{ Configuration } & Recall & Precision & $\begin{array}{c}\text { Ranking } \\
\text { Quality }\end{array}$ & Stability \\
\hline Random Matcher & $\mathbf{2 1 , 3} \%$ & $6,6 \%$ & 0,32 & 0,5 \\
\hline \hline DOCE (Base, $\boldsymbol{\pi}=\mathbf{5 0})$ & $\mathbf{6 2 , 7} \%$ & $28,6 \%$ & 0,51 & 4,3 \\
\hline DOCE (Text, $\boldsymbol{\pi}=\mathbf{5 0})$ & $\mathbf{7 3 , 0} \%$ & $35,8 \%$ & 0,57 & 5,3 \\
\hline DOCE (Text, $\boldsymbol{\pi}=\mathbf{1 0 0})$ & $\mathbf{7 9 , 0} \%$ & $37,3 \%$ & 0,57 & 6,2 \\
\hline
\end{tabular}

The DOCE algorithm performs significantly better than the Random Matcher across all measures and configurations as confirmed by t-tests $(\mathrm{p}<0.000001$ in all cases). DOCE can match more than $\mathbf{6 0 \%}$ more annotations than the random method. Furthermore, from the overall results the following conclusions can be drawn:

- The algorithm was able to detect almost $80 \%$ of cluster examples annotated by pedagogical experts (for the best configuration).

- The Precision result can be interpreted as meaning that only every third matching cluster is relevant. While this value is low, it is worth noting that the input maps contain 3.3 annotations on average; thus, fixing DOCE to always return the top 10, as we have done, will always produce relatively low Precision values.

- The Stability of the DOCE algorithm with respect to different models is relatively high. On average 6 clusters (for the best configuration) are in common when comparing two results sets produced by two different models against the same map, despite the fact that the models are often from discussions with different topics. 
- A more fine-grained analysis of the results showed that, on average, more than $60 \%$ of relevant clusters are exact matches (in comparison to $11 \%$ for the random matcher).

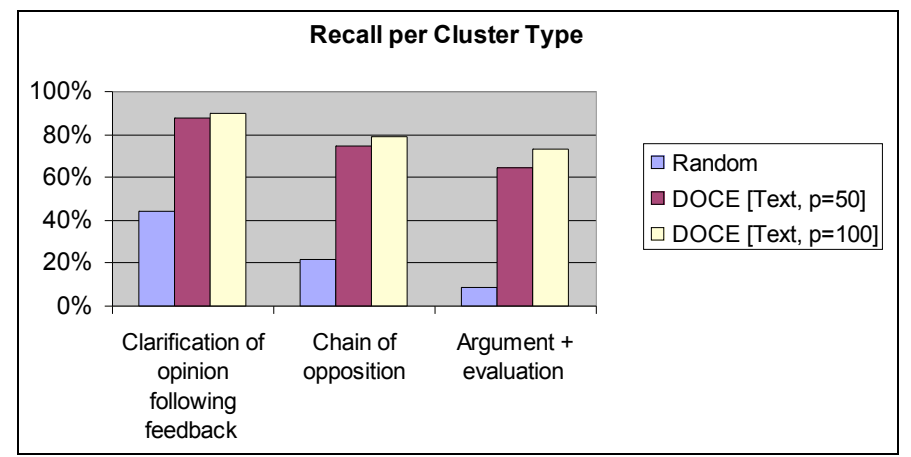

Fig. 3. Recall per cluster type

Finally, Fig. 3 shows the number of detected annotations in each map per cluster type. As can be seen, the algorithm delivered a relatively high Recall value for all three cluster types, significantly better than random, regardless of the $\pi$ value.

\section{Discussion and Further Work}

Although the initial results are quite promising, the algorithm was evaluated on a relatively small dataset. We had hoped the pedagogical experts on our project would be able to provide a much larger set of annotated maps, say hundreds, that could also be evaluated for inter-rater reliability. In fact, a single annotator provided all of the 74 annotations used in our evaluation. Despite this shortcoming, we argue that the dataset and annotations are sound and the evaluation meaningful because DOCE detects clusters that are similar to the provided models - in other words, the algorithm adapts to the "style" of the annotator. In practical terms, it seems unlikely that we will obtain a high level of inter-rater reliability for such an arduous and inexact task as identifying "meaningful" clusters, at least not without detailed specifications and extensive training of coders. On the other hand, note that many annotations were marked as borderline examples and could have negatively influenced the results of our experiments, yet we kept and used all of the annotations.

Currently, the pedagogical specialists are annotating additional maps and cluster types, and we plan further evaluation of the algorithm on a larger dataset. Additionally, we are working on integrating the algorithm into the Moderator's Interface in order to provide researchers with a tool for searching for more annotations. Another planned step is to experiment with using a set of models against one discussion map and then merging the results. Such an approach might improve DOCE's search accuracy. We will also tap our pedagogical experts' knowledge and perform experiments to customize the parameters of the algorithm. 
Our long-term goal is to obtain enough annotations to better understand the cluster types and develop an extended approach that can leverage domain knowledge. For example, an ML classifier may be used for filtering results produced by the DOCE algorithm.

\section{Conclusion}

Students in different classrooms around the world are using visual argumentation tools for e-discussions. In order to effectively moderate multiple, simultaneous discussions, a tool providing feedback to the teacher is required. The ARGUNAUT system is designed to help a teacher monitor the progress of multiple conversations through "Awareness Indicators" that display interesting events in the discussion.

In this work, we focused on analysis of segments of the discussion maps representing interaction patterns that are of pedagogical interest. Detection of such "clusters" of contributions is a complex task because the graph and text structure must be accounted for, the cluster types are imprecisely defined, and annotations are scarce.

We designed the DOCE algorithm to accept an example cluster and find similar clusters across different discussion maps. The method is an extension of the edit distance inexact graph matching algorithm and looks for subgraphs in the discussion maps that have the highest content similarity and lowest structural difference from an input model. The content similarity function accounts for discussion attributes, the text analysis performed by the TagHelper tool, and machine-learned classifications from the shape/pair-shape level.

We evaluated the algorithm on 27 actual discussion maps with 74 of the three most important clusters annotated by pedagogical experts. DOCE was able to detect almost $80 \%$ of the annotated clusters. We used all models in our evaluation, including ones from discussions with different topics and ones that were characterized as "borderline" examples. Furthermore, we compared the results with a random matcher, as there was no other "gold standard" algorithm available, and DOCE significantly outperformed this approach.

In sum, the experiments, although preliminary and on a limited dataset, have shown very promising results. However, deeper investigation and more extensive evaluation are planned. We intend to analyze the algorithm on a larger dataset and with more complicated clusters. We will also integrate the DOCE algorithm into the Moderator's Interface so it can help pedagogical experts define more annotations.

Acknowledgments. This work would not have been possible without project partners Rakheli Hever, Reuma De Groot, Maarten De Laat, Matthias Krauß, and Adam Giemza, as well as other members of the ARGUNAUT project team. The 6th Framework Program of the European Community, Proposal/Contract No. 027728, sponsored this research. 


\section{References}

1. Lingnau, A., Harrer, A., Kuhn, M., Hoppe, H.U.: Empowering teachers to evolve media enriched classroom scenarios. In: Research and Practice in Technology Enhanced Learning, vol. 2 (2), pp. 105-129. (2007)

2. Schwarz, B., De Groot, R: Argumentation in a changing world. In: International Journal of Computer-Supported Collaborative Learning, vol. 2, pp. 297-313. (2007)

3. Dillenbourg, P., Baker, H. P. M., Blaye, A., O'Malley, C.: The evolution of research on collaborative learning. In: Learning in humans and machines: Towards an interdisciplinary learning science, pp. 189-211. Elsevier/Pergamon, Oxford. (1995)

4. De Groot, R., Drachman, R., Hever, R., Schwarz, B.B., Hoppe, U., Harrer, A., De Laat, M., Wegerif, R., McLaren, B.M., Baurens, B: Computer Supported Moderation of EDiscussions: the ARGUNAUT Approach. In: Proceedings of the Conference on Computer Supported Collaborative Learning (CSCL-07), vol. 8, pp. 165-167. (2007)

5. McLaren, B. M., Scheuer, O., De Laat, M., Hever, R., De Groot, R., Rose, C. P.: Using Machine Learning Techniques to Analyze and Support Mediation of Student EDiscussions. In: Frontiers in Artificial Intelligence and Applications, pp. 331-338. IOS Press, Netherlands (2007)

6. Rosé, C., Wang, Y. C., Cui,Y., Arguello, J.,, Stegmann, K., Weinberger, A., Fischer, F.,: Analyzing Collaborative Learning Processes Automatically: Exploiting the Advances of Computational Linguistics in Computer-Supported Collaborative Learning. International Journal of Computer-Supported Collaborative Learning (in press).

7. Scheuer, O., McLaren, B.M.: Helping Teachers Handle the Flood of Data in Online Student Discussions. Submitted to the 9th International Conference on Intelligent Tutoring Systems to take place in Montreal, Canada, June 23-27, 2008 (submitted)

8. Kay, J., Maisonneuve, N., Yacef, K., \& Zaïane, O.: Mining patterns of events in students' teamwork data. In: Proceedings of the Workshop on Educational Data Mining at the 8th International Conference on Intelligent Tutoring Systems (ITS 2006), pp. 45-52. (2006)

9. Harrer, A., Hever, R., Ziebarth, S.: Empowering Researchers to Detect Interaction Patterns in e-Collaboration. In: Frontiers in Artificial Intelligence and Applications, vol. 158, pp. 503-510. (2007)

10. Zloof, M.M.: Query-by-Example: A Data Base Language. In: IBM Systems Journal, vol. 16, pp. 324-343. (1977)

11. Kolodner, J.: Case-based Reasoning, Morgan Kaufmann Publishers, San Francisco (1993)

12. Wong, A. K. C., You, M., Chan, S. C.: An algorithm for graph optimal monomorphism. In: Systems, Man and Cybernetics, IEEE Transactions on, vol. 20, pp. 628-638. (1990)

13. Tsai, W. H., Fu, K. S.: Error-correcting isomorphisms of attributed relational graphs for pattern recognition. In: IEEE Transactions on Systems, Man, and Cybernetics, vol. 9, pp. 757-768. (1979)

14. Messmer, B.T., Bunke, H.: Automatic Learning and Recognition of Graphical Symbols in Engineering Drawings. In: Selected Papers from the First International Workshop on Graphics Recognition, Methods and Applications, vol. 1072, pp. 123-134. (1996)

15. McLaren, B.M.: Extensionally defining principles and cases in ethics: An AI model. In: Artificial Intelligence, vol. 150, pp. 145-181. (2003)

16. Gregory, L., Kittler, J.: Using Graph Search Techniques for Contextual Colour Retrieval. In: Proceedings of the Joint IAPR International Workshop on Structural, Syntactic, and Statistical Pattern Recognition, vol. 2396, pp. 186-194. (2002)

17. Miksatko, J.: Using Machine Learning Techniques to Analyze and Recognize Complex Patterns of Student E-Discussions (M.Sc. Thesis). Charles University, Prague. (2007) 
What's in a Cluster? Automatically Detecting Interesting Interactions in Student E-Discussions

18. Borodin A., Roberts, G. O., Rosenthal, J. S., Tsaparas, P.: Link analysis ranking: algorithms, theory, and experiments. In: ACM Transactions on Internet Technology, vol. 5, pp. 231-297. (2005) 